\title{
Numerical Simulation of the Hydrodynamics of Some Sectors of the Prute River
}

\author{
GALINA MARUSIC ${ }^{1}$, VALERIU PANAITESCU2* \\ ${ }^{1}$ Technical University of Moldova, 168 Stefan cel Mare si Sfant BlvD., MD-2004, Chisinau, Republic of Moldova \\ 2 University Politehnica of Bucharest, 313 Splaiul Independentei, 060042, Bucharest, Romania
}

\begin{abstract}
This paper addresses issues related to the determination of hydrodynamics of aquatic systems, with the application of computational fluid dynamics (CFD) analysis. The importance of knowing the hydrodynamics of aquatic systems for solving water pollution problems, as well as their prevention, is discussed. A case study regarding the determination of the water flow characteristics using CFD for some sectors of the Prut river is presented.
\end{abstract}

Keywords: aquatic systems, numerical simulation, hydrodynamics, river, pollution, turbulent flow

Knowing the hydrodynamics of the aquatic systems has an importantrole in determining different processes, natural or artificial, that happen in the aquatic systems. The aquatic systems condition has been lately influenced by the human intervention. As a result, many artificial processes happen, such as: the dispersion of differenttypes of pollutants, water level decrease, the modification of the chemical-physical composition, etc.

Environmental pollution is a very important problem. A multiple ecologic pollution is attested when the values of the maximum admissible concentrations of different environmental factors are exceeded. In this case, the problem analysis concerning environmental loads, especially when these loads are admissible or not, has a big importance in determining the effects that follow [1].

Aquatic systems pollution has been a primordial problem in the last years and its effects negatively influence the human health. The problem appeared together with the socio-economic development, when a severe degradation of water quality was established. As a result of the human activity, the pollution of the aquatic systems with different types of substances (physical, chemical, biological) has been detected. This phenomenon has been stated in most countries. The consequences of these mentioned are the decrease of water reserve that can be used as drinking water source, the unbalance of ecosystems, the alarming increase of waste amount [24].

In order to reduce the negative effects of aquatic systems, CFD (computational fluid dynamics) has been lately used. It allows the modeling of different flow fluids processes, including the turbulent flow and determining the flowing parameters. The advantage of using CFD is in the possibility of getting detailed information referring to the simulated system [5 - 9].

\section{A research concerning the numerical simulation of the aquatic systems hydrodynamics}

The numerical simulation of the hydrodynamics is accomplished with the help of CFD, which uses the computing techniques and implies following the stages: modeling the geometry of the studied field, field meshing, model defining, setting the properties, establishing the initial and limit conditions, finding the solutions, results' analysis [5 - 9].

\footnotetext{
*Phone: 0214029441
}

In order to use the CFD when determining the aquatic systems hydrodynamics, mathematical and numerical modeling of these systems is necessary. Most flows, that happen, represent complex phenomena, and its turbulence, seems to be a very important component. Its characteristics are: irregularity, diffusive character, big Reynolds number, dissipative character, three dimensional character $[10,11]$.

According to Bradshow, turbulence is a threedimensional time-dependent motion in which vortex stretching causes velocity fluctuations to spread to all wavelengths between a minimum determined by viscous forces and a maximum determined by the boundary conditions of the flow [12].

The Navier-Stokes equation system is used to describe real flow fluid [7]. It consists of motion and continuity equations:

$$
\begin{aligned}
& \frac{\partial \mathbf{v}}{\partial t}+\mathbf{v} \nabla \mathbf{v}=\mathbf{f}-\frac{1}{\rho} \nabla p+v \Delta \mathbf{v}, \\
& \frac{\partial \rho}{\partial t}+\nabla(\rho \mathbf{v})=0
\end{aligned}
$$

where $\nabla$ is the Hamilton operator; $\Delta$ - Laplace operator; t-time; $v$-the kinematic viscosity; $\rho$-density; $p$-pressure; $\mathbf{v}$ - fluid velocity; f- external forces applied to the fluid.

It has been stated out that for most turbulent flows, the relation between the characteristic dimensions of big and small vortexes for turbulent vortexes spectrum is

$$
\frac{l}{\eta} \sim R e^{3 / 4},
$$

where $l$ represents the biggest scale of turbulent flow (integral scale); $\eta$-the smallest scale (Kolmogorov scale); $R e$-Reynolds number. Finding the exact solutions for the Navier-Stokes equations constitutes a big problem that cannot be solved, even if supercomputers would be used [7].

Numerical models are used for finding the solutions to the Navier-Stokes equations. The most used are: Direct Numerical Simulation (DNS), Reynolds Averaged (RANS), Large Eddy Simulation (LES), Detached Eddy simulation (DES) $[6,13]$.

The next stage in using CFD for turbulent flow modeling in river-type systems is finding the solutions to flow equations. The following software techniques of dynamic simulation are used: WASP (Water Quality Analysis 
Simulation Program), QUAL2E, ANSYS CFX (Computational Fluid Dynamics Software), GWLF (Generalized Watershed Loading Function), MONERIS (Modelling NutrientEmissions in River Systems), W QRRS (Water Quality for River Reservoir Systems), W MS (Watershed Modeling System), SMS (Surface-water Modeling System) etc. [14, 15].

For water flow modeling on the Prut River study area, the system of Navier-Stokes equations (4) and (5) was used, together with the continuity equation (6), which completely describe the dynamics of water in rivers in a turbulent regime $[16,17]$ :

$$
\begin{gathered}
h \frac{\partial u}{\partial t}+h u \frac{\partial u}{\partial x}+h v \frac{\partial u}{\partial y}-\frac{h}{\rho}\left(E_{x x} \frac{\partial^{2} u}{\partial x^{2}}+E_{x y} \frac{\partial^{2} u}{\partial y^{2}}\right)+g h\left(\frac{\partial H}{\partial x}+\frac{\partial h}{\partial x}\right)+ \\
+\frac{g u n^{2}}{\left(h^{1 / 6}\right)^{2}} \times\left(u^{2}+v^{2}\right)^{1 / 2}-\varsigma V_{a}^{2} \sin \psi+2 h \omega v \sin \varphi=0 \\
h \frac{\partial v}{\partial t}+h u \frac{\partial v}{\partial x}+h v \frac{\partial v}{\partial y}-\frac{h}{\rho}\left(E_{y x} \frac{\partial^{2} v}{\partial x^{2}}+E_{y y} \frac{\partial^{2} v}{\partial y^{2}}\right)+g h\left(\frac{\partial H}{\partial y}+\frac{\partial h}{\partial y}\right)+ \\
+\frac{g v h^{2}}{\left(h^{1 / 6}\right)^{2}} \times\left(u^{2}+v^{2}\right)^{1 / 2}-\varsigma_{a}^{2} \sin \omega+2 h \omega v \sin \phi=0 \\
\frac{\partial h}{\partial t}+h\left(\frac{\partial u}{\partial x}+\frac{\partial v}{\partial y}\right)+u \frac{\partial h}{\partial x}+v \frac{\partial h}{\partial y}=0
\end{gathered}
$$

In equations (4) - (6) $h$ is mean water depth $(\mathrm{m}), u$ local velocity in the $x$ direction $(\mathrm{m} / \mathrm{s}), \mathrm{v}$ - local velocity in $y$ direction $(\mathrm{m} / \mathrm{s}), t$ - time $(\mathrm{s}), \rho$ - density of water $\left(\mathrm{kg} / \mathrm{m}^{3}\right), g$ - gravitational acceleration $\left(\mathrm{m} / \mathrm{s}^{2}\right), E$ - coefficients of turbulent viscosity (Pa.s or kg/m/s), $H$ - share geodetic bed (m), $n$ - Manning coefficient of roughness, $\xi$ - empirical coefficient concerning the friction with air, $V_{a}$-wind speed $(\mathrm{m} / \mathrm{s}), \psi$-wind direction (degrees counterclockwise from the positive $x$-axis), $\omega$ - angular velocity of rotation of the Earth $(\mathrm{rad} / \mathrm{s}), \varphi$ - place latitude $[16,17]$ (Surface Water RMA2, 2011).

\section{Case study - determining the hydrodynamics on some sectors of the River Prut}

The Prut river constitutes an important water source, for the Republic of Moldova, as well as for Romania, its pollution having a major risk for the human health.

The hydrodynamics of the river has been modeled, according to Navier-Stokes equation system, as Reynolds. The hydrodynamics has been modeled according to turbulent flows. The characteristic flow sizes have been determined in the studied sector. These can be used for determining the concentration field of different types of pollutants.

Surface-water Modeling System (SMS) was used for numerical simulation, which is for modeling the process of surface waters. It has been made by the American specialists of Aquaveo companyand represents a program package that allows managing the process beginning with the import of topographic data and ending with visualizing and analyzing solutions [18].

The numerical models for determining the hydrodynamics have been made with the help of RMA2 (Resource Management Associates) program, from the SMS. Initially, the studied sector of the river Prutwas divided into three specific zones: the left bank, the right bank and the river. The properties for these zones have been established according to the options from RMA2 program. The method of Peclet number has been chosen. The recommended values for Peclet number are between 1540. In consequence, the coefficients of turbulent viscosity were calculated automatically, Peclet number, being Pe
$=20$. The values of the Manning coefficient of roughness have been introduced for each zone. This coefficient depends on the type of the material the bed river is made of. Considering, the bed river covered with sand and pebbles [19], the value $n=0.03$ has been used, taken from [20, 21].

A result of RMA2 program, the hydrodynamics of the studied sector in all finite elements was obtained, especially the velocity in $x$ and $y$ directions, as well as the resulting velocity. Also, the water depth in all finite elements was determined.

The numerical stability of RMA2 program was verified with the help of Froude number,

$$
F r=\frac{V}{\sqrt{g h}},
$$

where $V$ is water particles velocity; $g$ - gravitational acceleration; $h$ - the depth.

The RMA2 program can become unstable numerically if the Froude number is bigger than 0.6.

Four sectors of the Prut river have been studied: Sirauti, Braniste, Valea Mare, Cahul. In order to check the numerical stability of the RMA2 program, the Froude number has been determined for every scenario, concerning simulation, calibration and validation of the obtained numerical models (fig. 1).

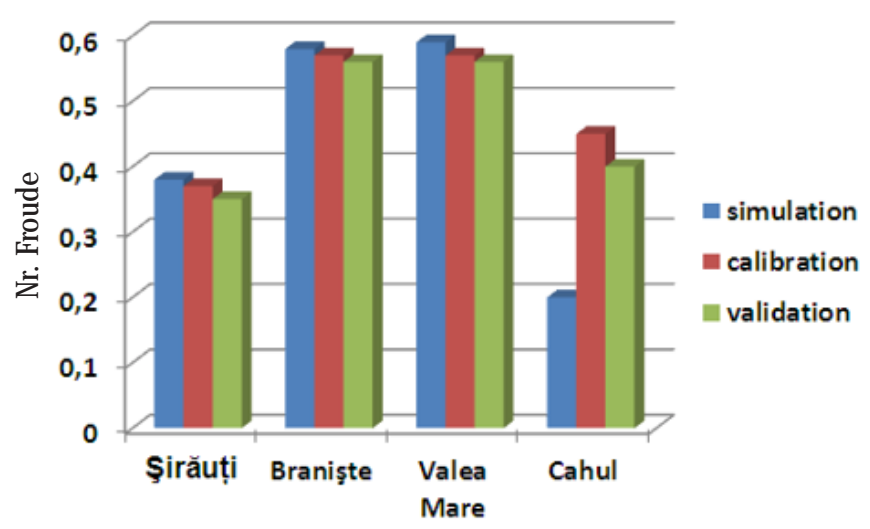

Fig. 1. The maximum values of the Froude number for the studied sectors

It can be noted out that the maximum value of the Froude number does not exceed the critical value of 0.6 .

For the purpose of numeric simulations, the studied sectors were discretized in finite elements directly in the SMS system. As a result of discretization, computing networks were obtained, which quality was verified using the Mesh Quality option from the SMS system. The obtained networks, as well as the sampling point, are presented in figure 2. The simulations have been completed in dynamic mode, for $12 \mathrm{~h}$, at intervals of $0.5 \mathrm{~h}$.

The results concerning the hydrodynamics of the studied sectors have been obtained after numerical simulations. The results of the numerical simulations with the help of RMA2 program are presented in figures 3-5. The local velocities in $x$ and $y$ directions have been determined in all the finite elements of the studied field geometry. For example, for the Prut river sector in the Sirauti locality, the results are presented in figure 3.

In figure 4 we can follow the distribution of the resulting velocity of water particles for the four studied sectors. The values of the resulting velocity are presented from the smallest to the biggest for three scenarios: simulation, calibration, validation. The values of the resulting velocity are the following: Sirauti $-0.076 \mathrm{~m} / \mathrm{s} \div 1.268 \mathrm{~m} / \mathrm{s}$; Braniste $-0.780 \mathrm{~m} / \mathrm{s} \div 3.014 \mathrm{~m} / \mathrm{s}$; Valea Mare $-0.377 \mathrm{~m} / \mathrm{s} \div 5.171$ $\mathrm{m} / \mathrm{s}$; Cahul $-0.404 \mathrm{~m} / \mathrm{s} \div 3.555 \mathrm{~m} / \mathrm{s}$. 


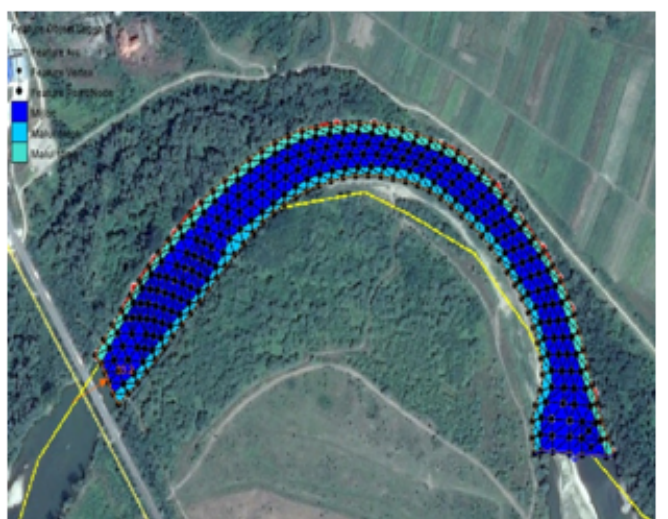

a)

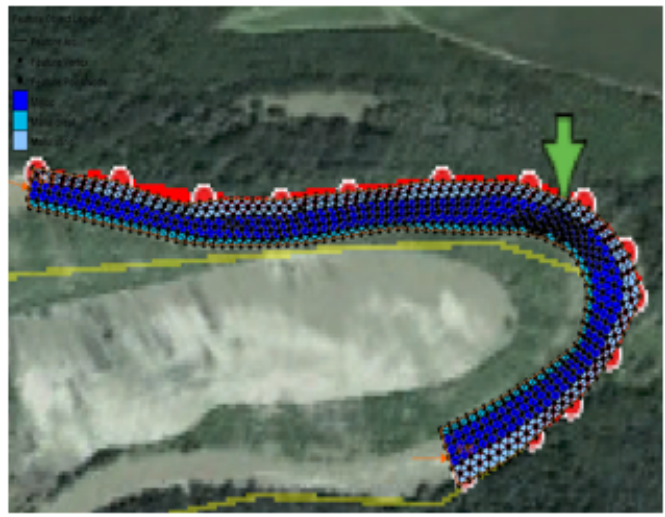

c)

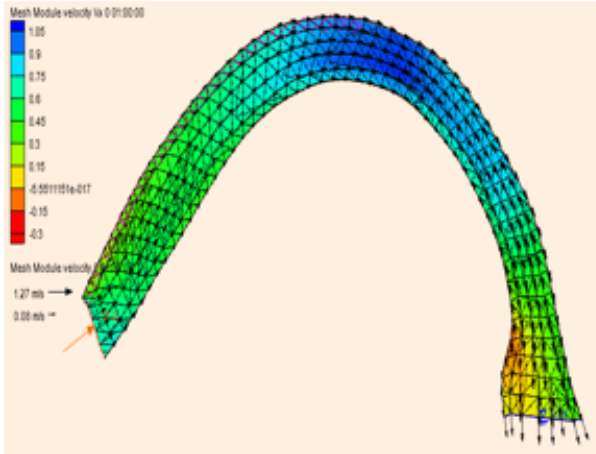

(a)

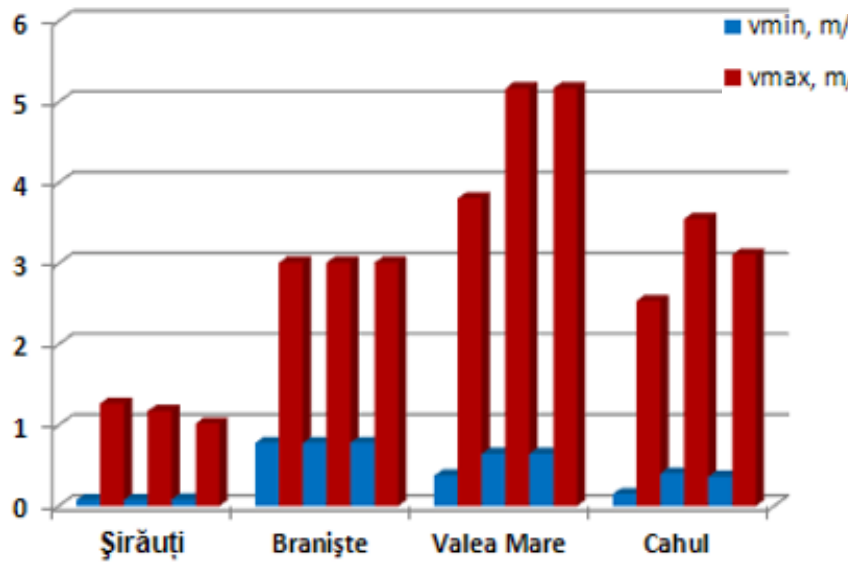

Fig. 4. Distribution of the resulting velocity

In figure 5 the depths variations of the studied sectors are presented, for three scenarios: simulation, calibration, validation. The values are the following: Sirauti $-0.319 \mathrm{~m}$ $\div 1.24 \mathrm{~m}$; Braniste $-2.001 \mathrm{~m} \div 2.59 \mathrm{~m}$; Valea Mare -0.463 $\mathrm{m} \div 5.687 \mathrm{~m}$; Cahul $-0.864 \mathrm{~m} \div 5.374 \mathrm{~m}$.

The obtained values concerning the hydrodynamics of the studied sectors can be used for simulating polluting
Fig. 2. Field of studied sector, discretized in finite elements: Sirauti (a), Braniste (b), Valea Mare (c), Cahul (d)
Fig. 3. Local velocities field in direction $x(a)$ and in direction $y(b)$, Sirauti 
The hydrodynamics for four sectors of the river Pruthas been obtained. The velocity field and water depth field have been established after numerical simulation of water flow in the studied sectors. The obtained results can be used for simulating pollution scenarios.

Determining the characteristic values of water flow with the help of CFD represents an efficient method in studying aquatic systems, as well as, solving problems concerning water pollution and preventing it.

The research results are, also, useful for assessing the evolution of water quality in aquatic systems.

\section{References}

1.J INESCU, V. V., MANEA, S.E., JINESCU, C., The Result of Loads Superposition Upon the Matter and Particularly Upon the Environment, Rev. Chim. (Bucharest), 68, no. 4, 2017, p. 656-665

2.MARUSIC, G., A study on the mathematical modeling of water quality in river-type aquatic systems, Journal W seas Transactions on Fluid Mechanics, Issue 2, Volume 8, April 2013, p. 80 - 89

3.OMER, I., Water Quality Assessment of the Groundwater Body RODL01 from North Dobrogea, Rev. Chim. (Bucharest), 67, no. 12, 2016, p. 2405-2408

4.CIRTINA, D., CAPATINA, C., Quality Issues Regarding the Watercourses from Middle Basin of J iu River, Rev. Chim. (Bucharest), 68, no. 1, 2017, p. 72-76

5.ANDERSON, Computational Fluid Dynamics for Engineers, Cambridge: University Press, 2012, p. 212

6.CHUNG, T.J., Computational Fluid Dynamics, Second edition, Cambridge: University Press, 2010, p. 1058

7.WILCOX, D., Turbulence Modeling for CFD, California: DCW Industries, 1994, $456 p$
8.CRISTEA, V., BAGIU, E., AGACHI, P., Simulation and Control of Pollutant Propagation in Somes River Using Comsol Multiphysics, Proceedings of the 20th European Symposium on Computer Aided Process Engineering - ESCAPE 20, Italy, 2010, p. 985-991

9. LAUNDER, B.E., SPALDING, D.B., The numerical computation of turbulent flows, Journal Computer Methods in Applied Mechanics and Engineering, 1974, vol. 3, Issue 2, p. 269-289

10.POPE, S., Turbulent Flows, Cambridge: University Press, 2003, p. 770

11.LOITSYANSKII, L.G., Mechanics of Liquids and Gases, (7th Ed.), 2003, p. 835

12.BRADSHAW, P., Introduction to turbulence and its measurement, Pergamon Press, 1971, p. 279

13.BLAZEK, J., Computational Fluid Dynamics for Engineers: Principles and Applications. Elsevier Science, 2005, p. 496

14.MARUSIC, G., Dynamic water quality simulation in river type system, Journal Academos, 3 (30), 2013, p. 39-44

15.MARUSIC, G., SANDU, I., FILOTE, C., SEVCENCO, N., CRETU, M.A. Modeling of Spacio - temporal Evolution of Fluoride Dispersion in River-type Systems, Rev. Chim. (Bucharest), 66, no. 4, 2015, p. 503506

16.GAVRILA, L., Transfer phenomena, Vol.1, Alma Mater, Bacau, Romania, 2000

17.*** Surface Water Modeling System - RMA2, US Army Engineer Research and Development Center, AquaVeo, USA, 2011

18.*** SMS Tutorials, SMS v.10.1.11, AquaVeo, 2011

19.YEARBOOK, Surface water quality status according to hydrobiological elements on the territory of the Republic of Moldova in 2012, Chisinau, 2013, p.145

20.HEC-RAS River Analysis System, Hydraulic Reference Manual, US Army Corps of Engineers, 2010, p. 411

21.*** Surface Water Modeling System - RMA4, US Army Engineer Research and Development Center, AquaVeo, USA, 2011.

$\overline{\text { Manuscript received: } 18.10 .2017}$ 